\title{
Clinicopathologic retrospective analysis of blastic plasmacytoid dendritic cell neoplasms
}

\author{
Agnieszka Owczarczyk-Saczonek ${ }^{1}$, Małgorzata Sokołowska-Wojdyło², Berenika Olszewska², Marta Malek ${ }^{3}$, \\ Aleksandra Znajewska-Pander ${ }^{1}$, Anna Kowalczyk ${ }^{4}$, Wojciech Biernat', Grażyna Poniatowska-Broniek ${ }^{6}$, \\ Wanda Knopińska-Posłuszny ${ }^{7}$, Zygmunt Kozielec ${ }^{6}$, Roman Nowicki², Waldemar Placek ${ }^{1}$
}

\author{
1Department of Dermatology, Sexually Transmitted Diseases and Clinical Immunology, University of Warmia and Mazury, Olsztyn, \\ Poland \\ ${ }^{2}$ Department of Dermatology, Venereology and Allergology, Medical University of Gdansk, Gdansk, Poland \\ ${ }^{3}$ Dermatology Outpatient Clinic, UCK Medical University of Gdansk, Gdansk, Poland \\ ${ }^{4}$ Department of Oncology and Radiotherapy, Medical University of Gdansk, Gdansk, Poland \\ ${ }^{5}$ Department of Pathomorphology, Medical University of Gdansk, Gdansk, Poland \\ ${ }^{6}$ Department of Pathomorphology, University of Warmia and Mazury, Olsztyn, Poland \\ ${ }^{7}$ Department of Hematology, Hospital of the Ministry of Internal Affairs and Administration, Olsztyn, Poland
}

Adv Dermatol Allergol 2018; XXXV (2): 128-138

DOI: https://doi.org/10.5114/ada.2017.72269

\begin{abstract}
Blastic plasmacytoid dendritic cell neoplasm (BPDCN) is one of the aggressive rare hematopoietic malignancies with predilection to the skin, primarily found in adults. The precise incidence of BPDCN is difficult to estimate due to constantly changing nomenclature and lack of precise defining criteria prior to the 2008 WHO classification system. There are not many cases described in the literature, what makes the diagnostic process challenging. Skin lesions such as erythematous infiltrates and nodules are usually the first manifestation of the disease. Therefore, in doubtful diagnostic cases, dermatologists should perform histopathological and immunohistochemistry examinations along with hematological and oncological cooperation, as early diagnosis and appropriate treatment is essential for improvement of the disease course. This analysis, despite the small number of patients may provide useful information on the clinical and histopathological features of this rare malignancy.
\end{abstract}

Key words: blastic plasmacytoid dendritic cell neoplasm, CD4, CD56.

Blastic plasmacytoid dendritic cell neoplasm (BPDCN) is a very rare condition, which represents $0.44 \%$ of all hematologic malignancies, $0.7 \%$ of cutaneous lymphomas and $6.3 \%$ of the NK cell line (Japanese data) [1-4]. Only 100 cases have been reported worldwide [5]. Skin lesions are the first clinical symptoms of the disease in more than $90 \%$ of cases. They are characterized by single or multiple infiltrations and violet tumors without tendency to ulceration. The mucosae involvement is observed rarely [3, 4, 6-9]. The course of the disease is usually aggressive. At the time of diagnosis, the disease is usually already generalized, with involvement of the lymph nodes, bone marrow, peripheral blood and the development of acute myeloid leukemia (AML) [10, 11]. Cytostatic treatment, in some cases associated with ra- diation, usually only slightly modifies the course of the disease [3].

The malignancy was initially described in 1994 as a CD4 - positive lymphoma with high CD56 expression. It was referred to as "blastic natural killer (NK) cell lymphoma” or "agranular CD4 + NK cell leukemia” [2, 12, 13]. However, following the confirmation that this neoplasm is derived from plasmacytoid dendritic cell type II precursors (pDCs), it was renamed as blastic plasmacytoid dendritic cell neoplasm and categorized under a subtype of acute myeloid leukemia according to the $2008 \mathrm{WHO}$ Classification of Tumors of Hematopoietic and Lymphoid Tissues [2, 14].

Plasmacytoid dendritic cells constitute less than $0.4 \%$ of peripheral blood mononuclear cells. They are increased

Address for correspondence: Agnieszka Owczarczyk-Saczonek MD, PhD, Department of Dermatology, Sexually Transmitted Diseases and Clinical Immunology, University of Warmia and Mazury, 30 Aleja Wojska Polskiego St, 10-595 Olsztyn, Poland, phone: +48 $601057800,+488967866$ 70, fax: +48 8967866 75, e-mail: aganek@wp.pl Received: 1.03.2017, accepted: 1.04.2017. 
in a variety of pathological conditions, such as autoimmune diseases, non-Hodgkin lymphomas and cancers [5].

Microscopically, the disease is characterized by medium-sized monomorphic neoplastic cells resembling leukemic infiltrates with blastic appearance, sometimes elongated and twisted $[8,15]$. The BPDCN phenotype is CD4, CD56 and CD123, often TdT + MPO- $[10,16]$. The genetic analysis confirms presence of the TCR and IGH genes in germline configuration. In single cases, TCR gene rearrangements occur. Complex karyotypes and chromosome anomalies are observed [8].

In addition to the initial presentation in the skin, the disease involves extracutaneous organs: $40-50 \%$ of patients display lymph node involvement, $60-90 \%$ of patients show bone marrow and peripheral blood involvement, and the spleen is involved only in $20 \%$ of cases [ 6 , 10]. In contrast, the central nervous system involvement is observed mainly in cases of disease recurrence. Group B symptoms, such as fever, night sweats and weight loss are rare. Pancytopenia, and most commonly thrombocytopenia, is often present in laboratory results $[6,17]$. Leukemia is a common feature for the end stage of disease or relapse of the disease, $10-20 \%$ of BPDCN cases are accompanied or may progress to acute myeloid leukemia $[6,17]$.

Case 1 (72-year-old female patient). The first lesion presented with red-brown induration that appeared on the skin of the trunk in January 2012. In March, skin biopsy suggested the T-cell pseudolymphoma. Due to the fact that new skin lesions appeared, in May 2012 the patient was admitted to the Department of Dermatology, Venereology and Allergology, Medical University of Gdansk to diagnose indurated erythematous lesions and nodules on the trunk and face (Figure 1). During hospitalization, the patient complained of itching, burning sensations and skin pain. In addition, the patient experienced productive cough with white-tinted content, but no weight loss, night sweats and high temperatures were observed.
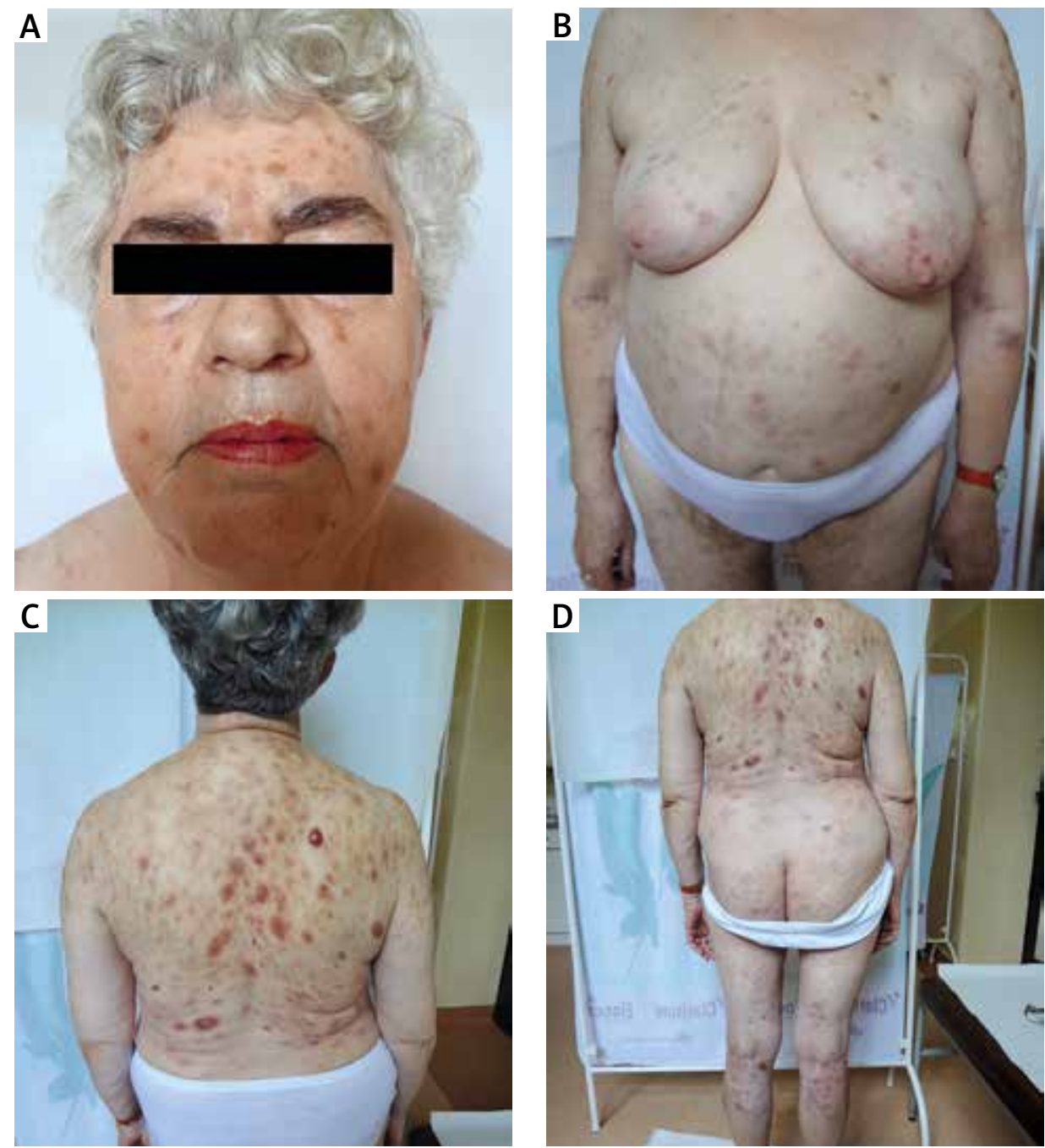

Figure 1. Cutaneous manifestation of BPDCN: bruise-like nodular form (A, B, C, D) 
The patient had a history of hypertension, gastroesophageal reflux, a history of surgery of the thyroid gland (1987), hysterectomy because of fibroids (1972), and cholecystectomy (1991). The family medical history: the patient's father had duodenal cancer, mother had uterine cancer and sister had colon cancer.

Some abnormalities were found in laboratory examinations: leukopenia (WBC $3.07 \mathrm{G} / \mathrm{l}$ ) with neutropenia (1.24 G/l), following normal HGB concentration (13.4 G/l) and normal platelets concentration (PLT 206 G/I). The repeated skin biopsy confirmed the diagnosis of blastic plasmacytoid dendritic cell neoplasm: diffuse infiltration of neoplasm cells in the skin, without epidermotropism, with cell expression: CD3-CD4+CD56+TIA 1- granzyme B-CD20-CD8-TdT-/+ (15\%) CD34-index Ki67 70\%. The bone marrow biopsy showed no signs of blastic plasmacytoid dendritic cell neoplasm infiltration.

Chest X-ray and abdominal ultrasonography were within normal limits. However, the computed tomography $(\mathrm{CT})$ revealed uncharacteristic lymph nodes along the iliac vessels and retroperitoneal flat, in the right armpit single, pathological lymph nodes up to $23 \times 33 \mathrm{~mm}$, segmental thickening of the skin and infiltration in subcuta-
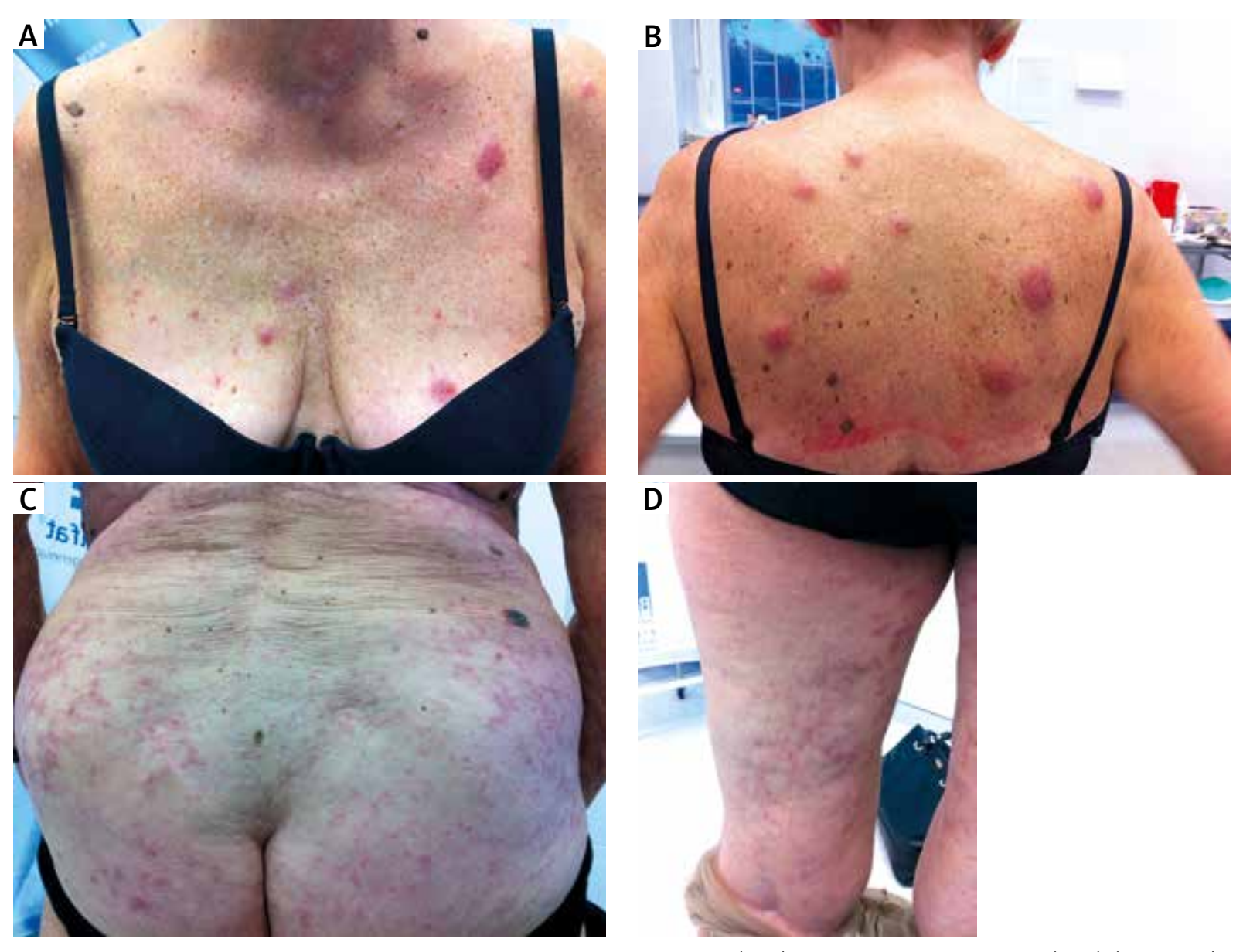

Figure 2. Cutaneous manifestation of BPDCN: bruise-like nodular form (A, B) and disseminate lesions (C, D) (Nov 2014) neous adipose tissue within the chest. Moreover, small uncharacteristic nodules were found in the upper lobe of the right lung, probably in the course of the underlying disease.

The patient was referred for further oncological diagnosis and treatment, however she did not show up.

Case 2 (60-year-old female patient). A 60-year-old woman presented with a 3-week history of numerous violet nodules on the back and single nodules on the cleavage without itching. They were accompanied by disseminated papules, merging on the trunk and lower extremities. Skin lesions were previously misdiagnosed as drug-induced skin reaction to the antipyretic drug that the patient had taken 2-3 days before (Figure 2).

The skin biopsy showed a diffuse monomorphic infiltration of the dermis with large lymphoid cells with irregular nuclei with a clear nucleoli and phenotype: CD3-CD4+CD5+CD7-CD8-CD10-PD1-CXCL13-MPOCD34-CD20-CD56+ ALK- Granzyme B1-TIA1-CD43+ CD68+ (dot) TdT-Ki67 index of 18\%. Blastic plasmacytoid dendritic cell neoplasm was diagnosed (Figures 3, 4).

Some abnormalities were found in laboratory examinations: increased white blood cell concentration 
(WBC $45.04 \mathrm{G} / \mathrm{l})$ with abnormalities in peripheral blood: segments $23.5 \%$, sticks $5 \%$, metamyelocytes $5 \%$, myelocytes $5 \%$, promyelocytes $2 \%$, blasts $15.5 \%$, basophils $0.5 \%$, eosinophils $1.5 \%$, monocytes $27 \%$, conoid cells $15 \%$, lymphocytes $15 \%$. In addition, medium-weight to heavy, neutrophils' granules were present in leukocytes, polychromatic (RBC) (+), anisocytosis (RBC) (+), and platelets of negligible anisocytosis, erythroblasts - relative value of $0.8 \%$. Increased $\beta_{2}$-mikroglobulin 5.289 (standard $2.5 \mathrm{mg} / \mathrm{dl}$ ), LDH 460 (normal range: 120-230 U/I) was observed. The patient was referred to the Department of Hematology and Transplantation UCK, Medical University of Gdansk, where she received cytoreductive treatment twice: ARAC + Mitoxantrone (2 and 8 January 2015).
A

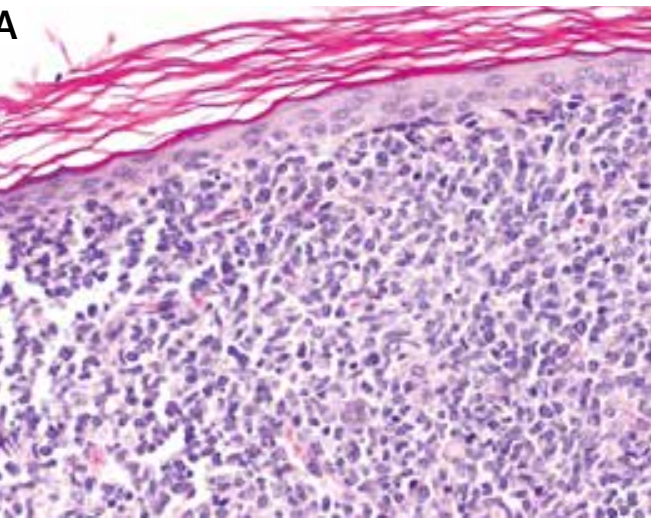

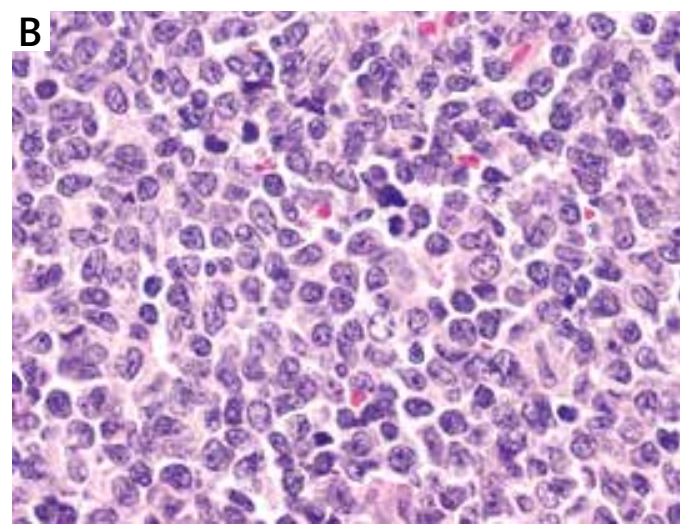

Figure 3. Blastic plasmacytoid dendritic cell neoplasm. Diffuse infiltration of the skin below the attenuated epidermis (A) is composed of monomorphous infiltration of medium sized blast cells with irregular nuclei, fine chromatin and one to several small nucleoli (B)
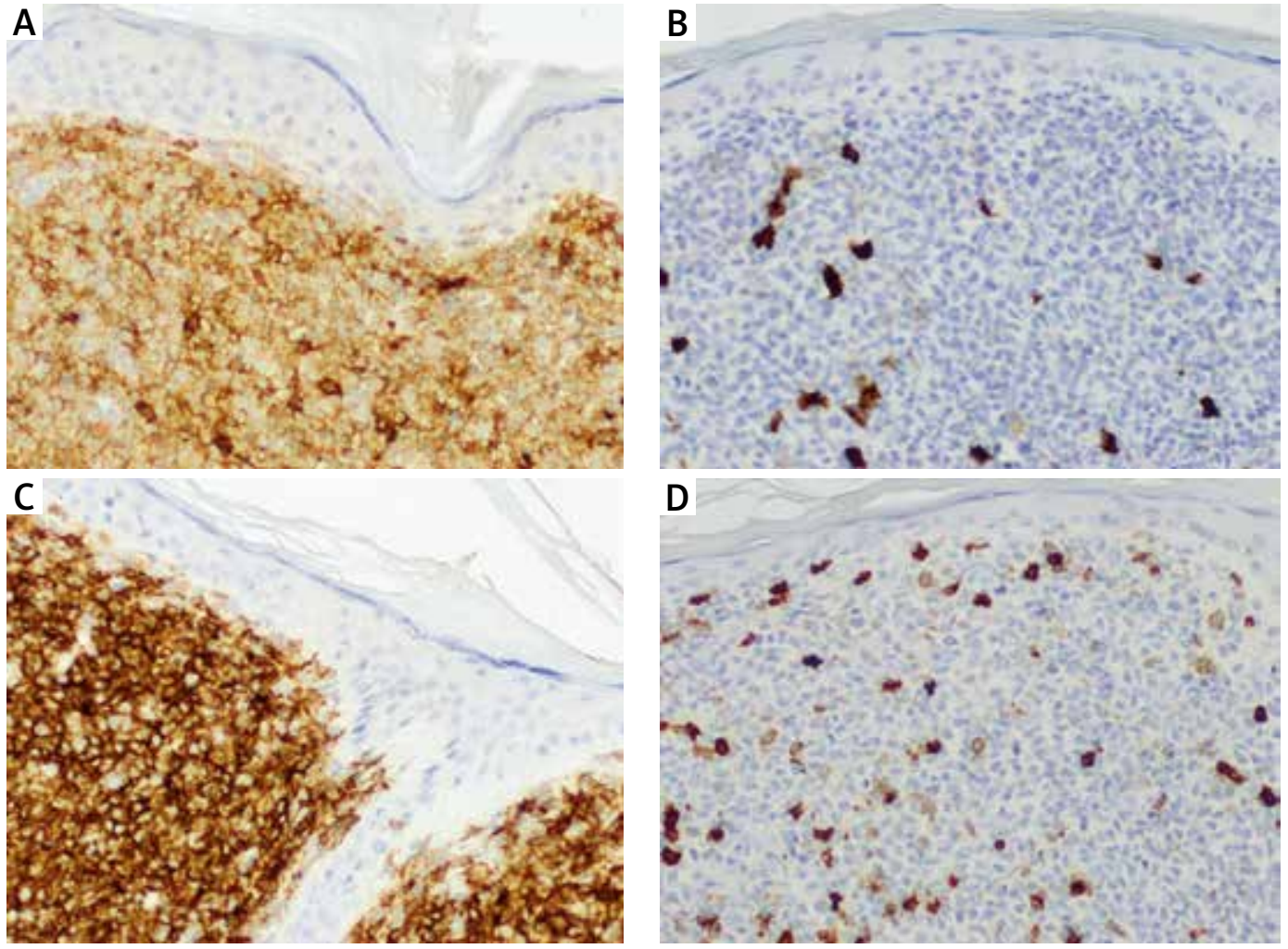

Figure 4. Blastic plasmacytoid dendritic cell neoplasm. The typical phenotype of the tumor with CD4 (A) and CD56 (C) expression and lack of CD8 (B) and CD3 (D) 
During further follow-ups in the Outpatient Dermatological Clinic progression of the disease was observed. New erythematous infiltrations of the skin accompanied by severe pruritus appeared. Intensity of pruritus increased with leukocytosis (Figure 5). The pruritus severity according to the patient was 10 (scale range: 0-10). The patient was treated with desloratadine p.o. to reduce itching. Laboratory tests revealed leukocytosis (WBC 53.7 G/l), HGB 10.7 and LDH 537.

Cytologic and immunophenotypic examination of bone marrow (4 July 2015) revealed acute myelomonocytic leukemia. The peripheral blood film showed (04/05/2015): segments 9.3\%, sticks 1.8\%, metamyelo- cytes $1.9 \%$, myelocytes $4.6 \%$, promyelocytes $1.9 \%$, blasts $51.8 \%$, eosinophils $0.9 \%$, monocytes $6.5 \%$, lymphocytes $21.3 \%$, neutrophils medium-weight granules in leukocytes, single erythrocytes, lacrymocytes, anisocytosis (RBC)++, platelets of normal morphology and NRBC 0.14, erythroblasts - relative value of $0.7 \%$.

During hospitalization (12 May 2015) in the Department of Hematology and Transplantation, the patient received etoposide. Pruritus severity decreased to 4, however skin lesions did not response to treatment. $A$ decrease in leukocytosis (WBC $18.1 \mathrm{G} / \mathrm{l}$ ) and LDH to 349 was observed. Afterwards the patient received cytoreductive treatment with cytarabine (100 mg i.v. from 26 June to
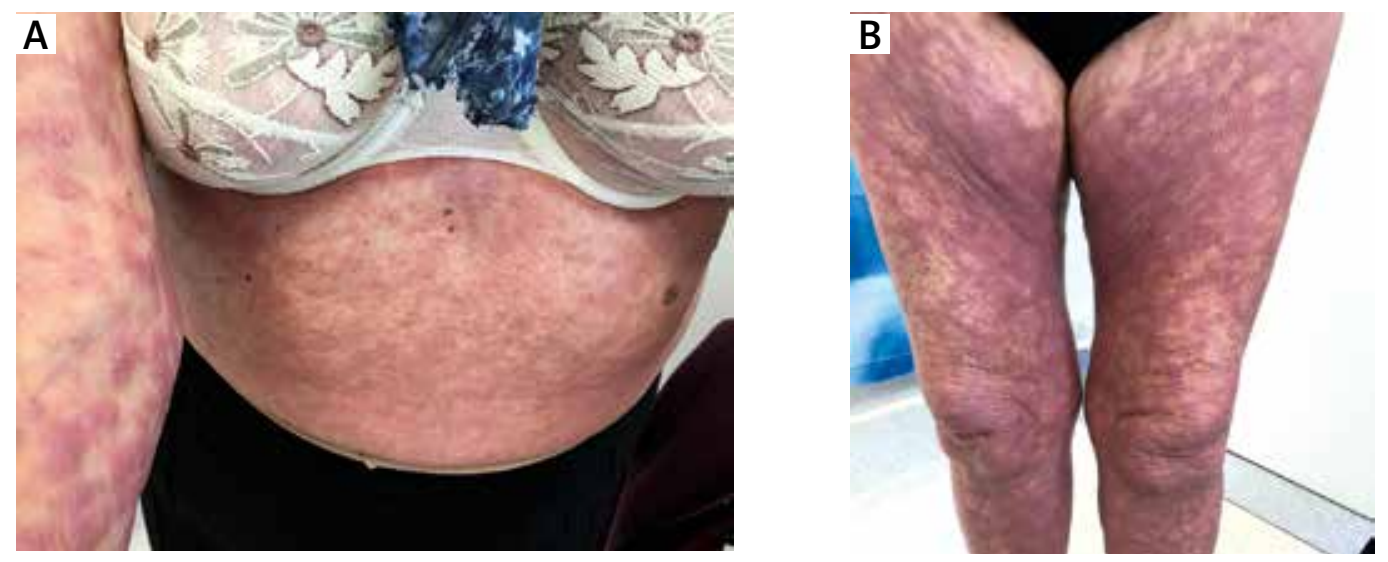

Figure 5. Exacerbation of cutaneous lesions in the form of disseminate exanthema (A, B) Feb 2015

A

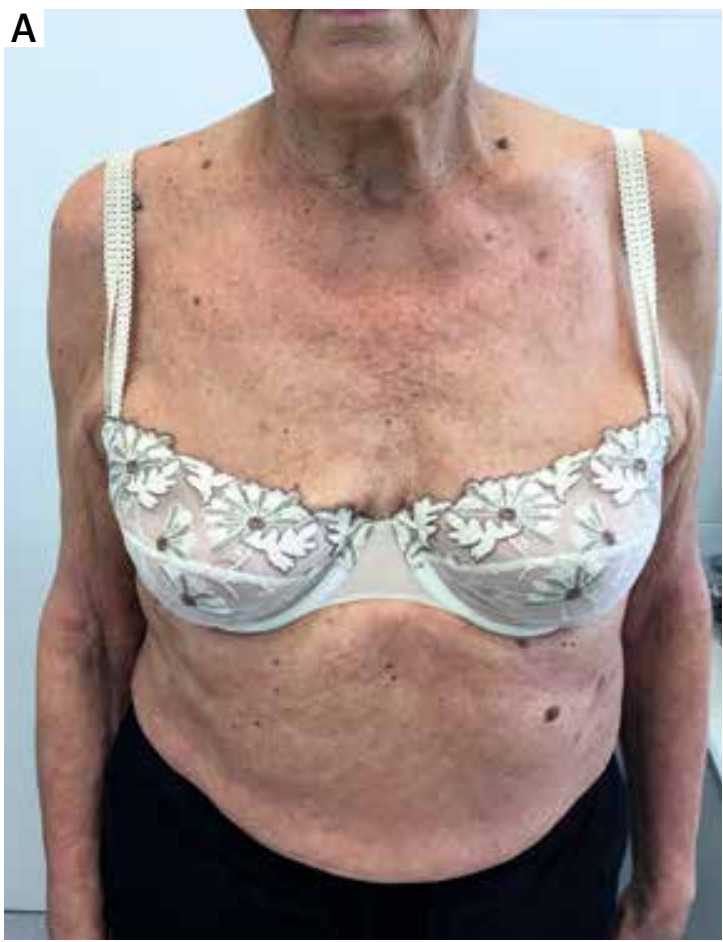

B

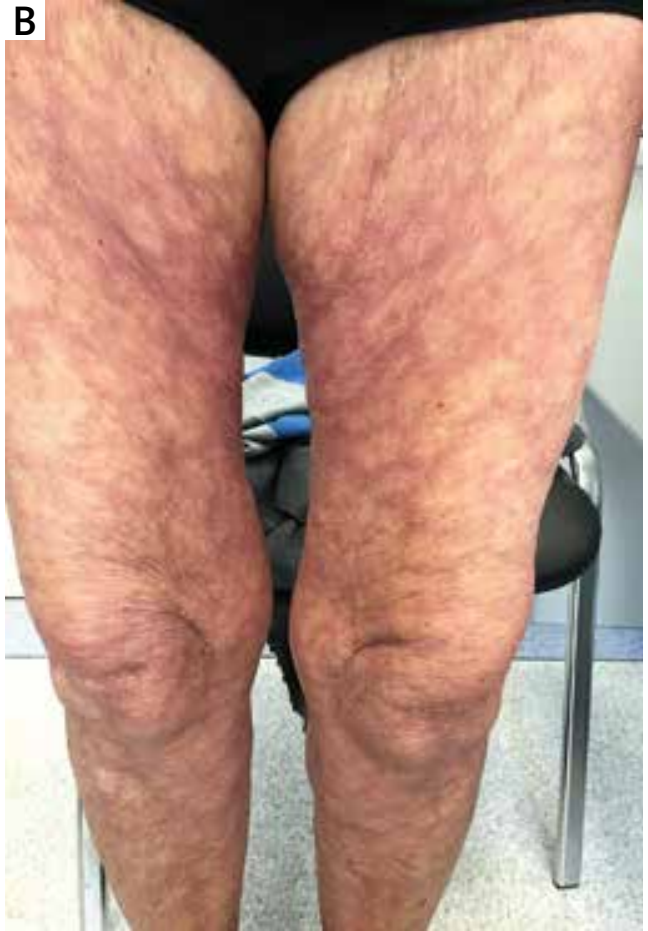

Figure 6. Patient after therapy - remission of skin lesions (July 2015) 
8 July 2015), which resulted in partial resolution of cutaneous lesions and pruritus (Figure 6). In the initial phase of regeneration, blast cells appeared in the peripheral blood film, however neither the patient's condition nor the skin condition got worse. After the treatment, leukocyte concentration was $23 \mathrm{G} / \mathrm{l}$ and peripheral blood film presented: $21.4 \%$ monocytes, sticks $5.5 \%$, segments $18.9 \%$, lymphocytes $19.9 \%$, blasts $15.4 \%$, erythroblasts $1.5 / 100$, platelets of negligible anisocytosis, the presence of neutrophils granules, anisocytosis (RBC), basophils $0.5 \%$, eosinophils $2 \%$, lacrymocytes, metamyelocytes $8.9 \%$, myelocytes $4.5 \%$, promyelocytes $3 \%$. The patient was subsequently qualified for the bone marrow transplant.

Case 3 (63-year-old female patient). A 63-year-old woman with rheumatoid arthritis was admitted to the
Department of Dermatology, Sexually Transmitted Diseases and Clinical Immunology, University of Warmia and Mazury on 3 March 2014 in order to diagnose solitary nodules (1-3 cm in diameter) on the right leg accompanied by swelling and presence of enlarged lymph nodes in the right groin (Figure 7). The first skin lesions occurred in September 2013; in December 2013 she was admitted to the Department of Surgery Hospital in Lidzbark where deep venous thrombosis was excluded and anti-inflammatory treatment (Nimesil, Cyclonamine) was performed. The treatment did not result in improvement of skin lesions. In February 2014, new nodules appeared on the leg.

Laboratory examinations revealed elevated LDH 564 $\mathrm{U} / \mathrm{I}$. Doppler ultrasound of veins and arteries of lower ex-
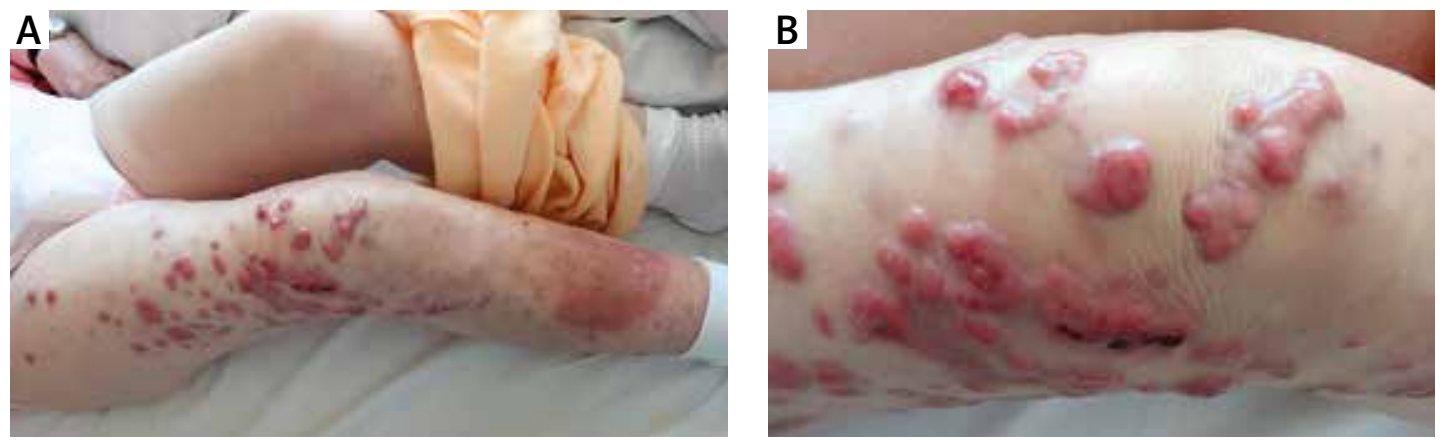

Figure 7. Numerous nodules with hemorrhagic component (A, B)
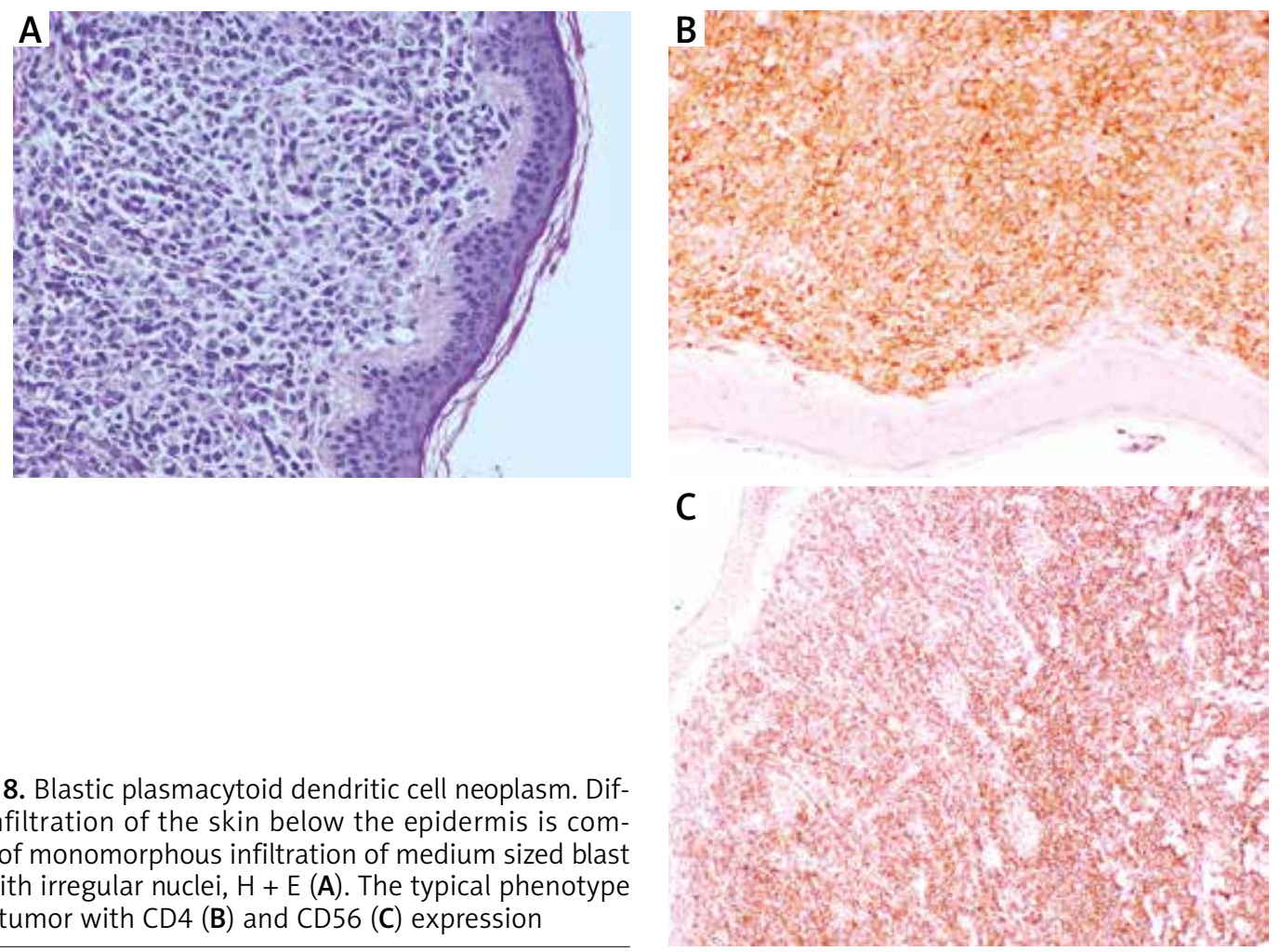

Figure 8. Blastic plasmacytoid dendritic cell neoplasm. Diffuse infiltration of the skin below the epidermis is composed of monomorphous infiltration of medium sized blast cells with irregular nuclei, $\mathrm{H}+\mathrm{E}(\mathrm{A})$. The typical phenotype of the tumor with CD4 (B) and CD56 (C) expression 
tremities, abdominal ultrasound, chest $\mathrm{X}$-ray results were within normal limits.

A punch biopsy of the nodule confirmed the diagnosis of blastic plasmacytoid dendritic cell neoplasm. The biopsy showed a diffuse monomorphic medium-sized blast cells infiltration of the dermis with the following immunophenotype: CD4+, CD56+, LCA+, CD43+, BCL2+, CD33+, CD68+ ("dot" cytoplasmic pattern), Ki67+++ (approximately $85 \%$ of cells), CD138+/- (very weak “dot” cytoplasmic pattern in many cells), TdT- (negative nuclear pattern, but the majority of cells displayed a weak "dot" cytoplasmic pattern), EMA-/+ (very weak "dot" pattern in some cells), MPO- (in some cells a cytoplasmic "dot" pattern was positive), CD117-, CD34- (positive reaction in cells), CD13- (positive reaction in single cells), CD20-, cyclin D1-, CD10-, BCL6-, MUM1-, CD3-, CD2-, CD5-, CD7-, CD30-, ALK1-, MelanA-, S-100-, CKAE1/AE3-, Chromogranin- (Figure 8).

Due to BPDCN diagnosis, the patient was urgently referred to the Department of Hematology, Hospital of the Ministry of Internal Affairs and Administration in Olsztyn, in order to begin chemotherapy.

Laboratory examinations revealed benign anemia and elevated LDH. CT revealed mediastinal lymph nodes up to $15 \mathrm{~mm}$, and in the abdominal cavity: the lymph nodes up to $20 \mathrm{~mm}$ surrounding the left common iliac artery on the right side up to $17 \mathrm{~mm}$, lymph nodes surrounding the right external iliac artery up to $20 \mathrm{~mm}$, lymph nodes on the left side up to $12 \mathrm{~mm}$, right inguinal lymph nodes up to $35 \mathrm{~mm}$ merging into conglomerates, on the left side up to $9 \mathrm{~mm}$.

The stage of the disease was determined as IVA, IPI 4. On 18 April 2014, the patient began treatment with the chemotherapy regimen: CHOEP. The patient received 6 cycles of chemotherapy (until August 2014). The par- tial remission within the skin (Figure 9) and complete remission within lymph nodes was obtained, which was confirmed by CT. Two weeks after chemotherapy, rapid progression of the disease was observed. Due to resistance to previous treatment, palliative radiotherapy was performed. Unfortunately, the patient's condition deteriorated. After 3 weeks the patient died.

Case 4 (79-year-old male patient). A 79-year-old man was admitted to the Department of Dermatology, Venereology and Allergology, Medical University of Gdansk on 4 July 2015 due to indurated erythematous lesions and nodules localized on the scalp, face and trunk, which occurred four weeks earlier (Figure 10). The patient denied itching and fever.

The diagnostic tests revealed some abnormalities: elevated $\beta_{2}$-microglobulin $3.38 \mathrm{mg} / \mathrm{l}$, monocytosis $1.8 \mathrm{G} / \mathrm{l}$, the presence of immature granulocytes $0.8 \%$ and reduced platelets concentration $116 \mathrm{G} /$. Chest X-ray was within normal limits, while abdominal ultrasound revealed a cyst in the liver $8 \times 10 \mathrm{~mm}$, and a structure in the urinary bladder that could account for diverticulum. Computed tomography of the abdomen confirmed the ultrasound results. Colonoscopy was performed due to the positive result for blood in the stool test. We observed hemorrhoids and numerous polyps that corresponded to adenomata tubularia coli duo cum dysplasia gradus minoris epithelii glandularis in histopathological examination. Skin biopsy revealed normal epidermis, dermal fibrosis with diffuse infiltration of lymphoid cells (CD56+, CD163+, CD3, CD4+, CD8+, CD30-, CD34, MPO-, CD117-, CD7-, CD5-, ALK-, CD1a-) and weak epidermotropism, which support the diagnosis of blastic plasmacytoid dendritic cell neoplasm.

During hospitalization, despite the lack of systemic treatment partial spontaneous remission of the disease
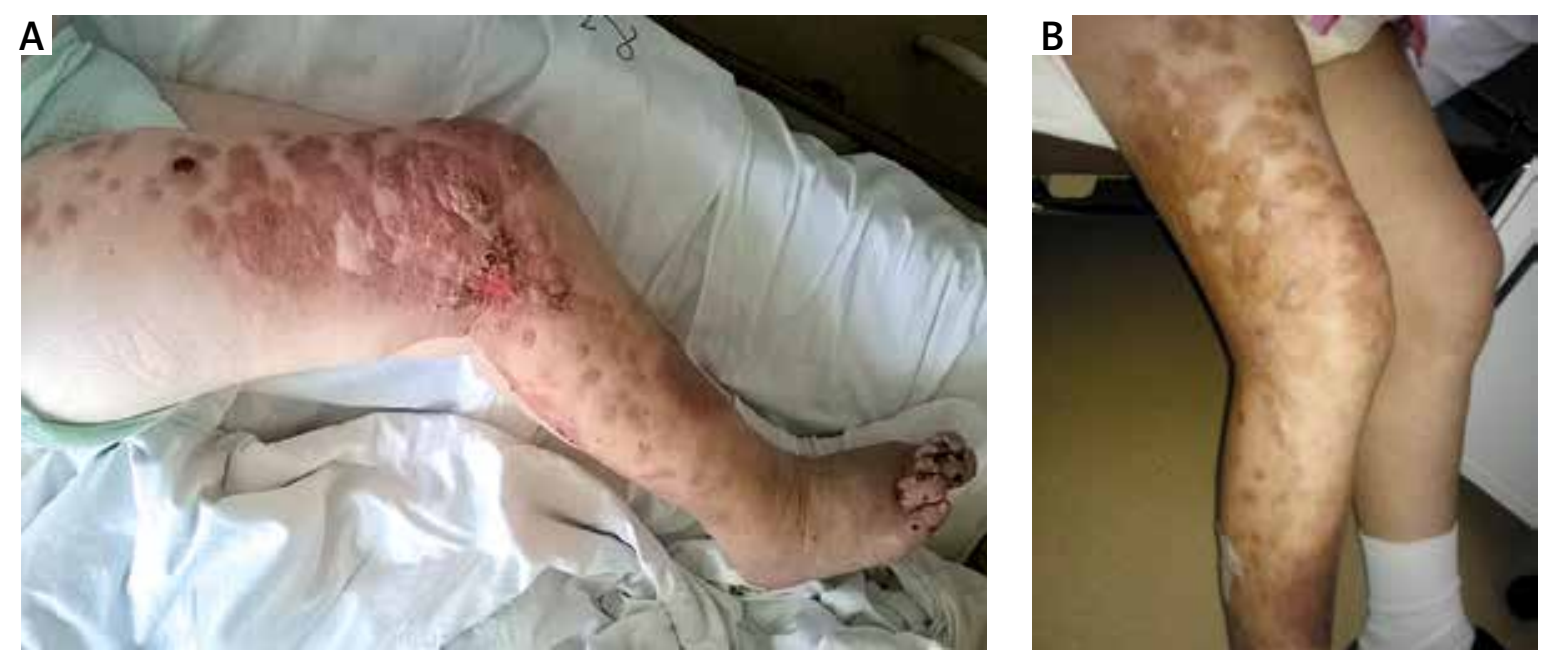

Figure 9. The clinical appearance 2 days after the first cycle of chemotherapy, April 2014 (A) and after five cycles of chemotherapy, September 2014 (B) 
was observed. The patient was referred for further oncological diagnosis and treatment (Figure 11).

Blastic plasmacytoid dendritic cell neoplasm (BP$D C N$ ) is a rare hematologic neoplasm. The precise incidence of BPDCN is difficult to estimate due to constantly changing nomenclature and lack of precise defining criteria prior to the 2008 WHO classification system [14].

Blastic plasmacytoid dendritic cell neoplasm has been described in all age groups, but the majority of patients are adults and the average age is 60 to 70 years (range: $8-103$ years) $[2,5,16,18]$. The condition is rarely diagnosed in children, so far only 36 cases have been reported [19].

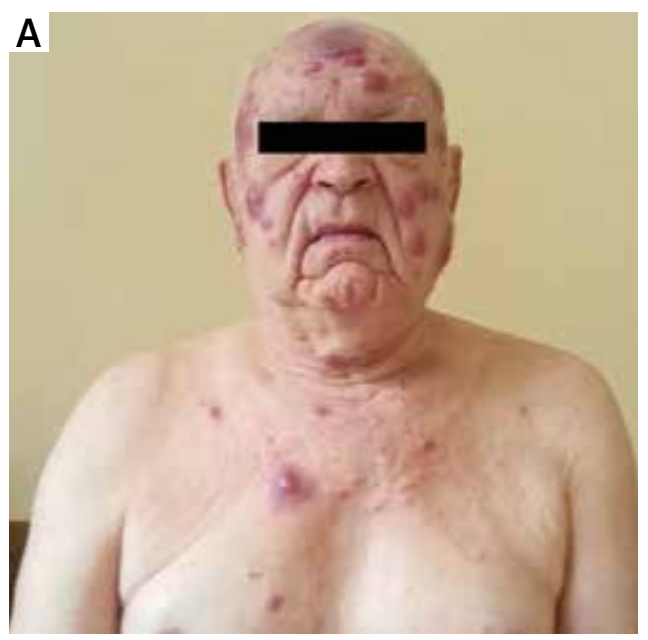

Figure 10. The bruise-like nodular form (A, B)
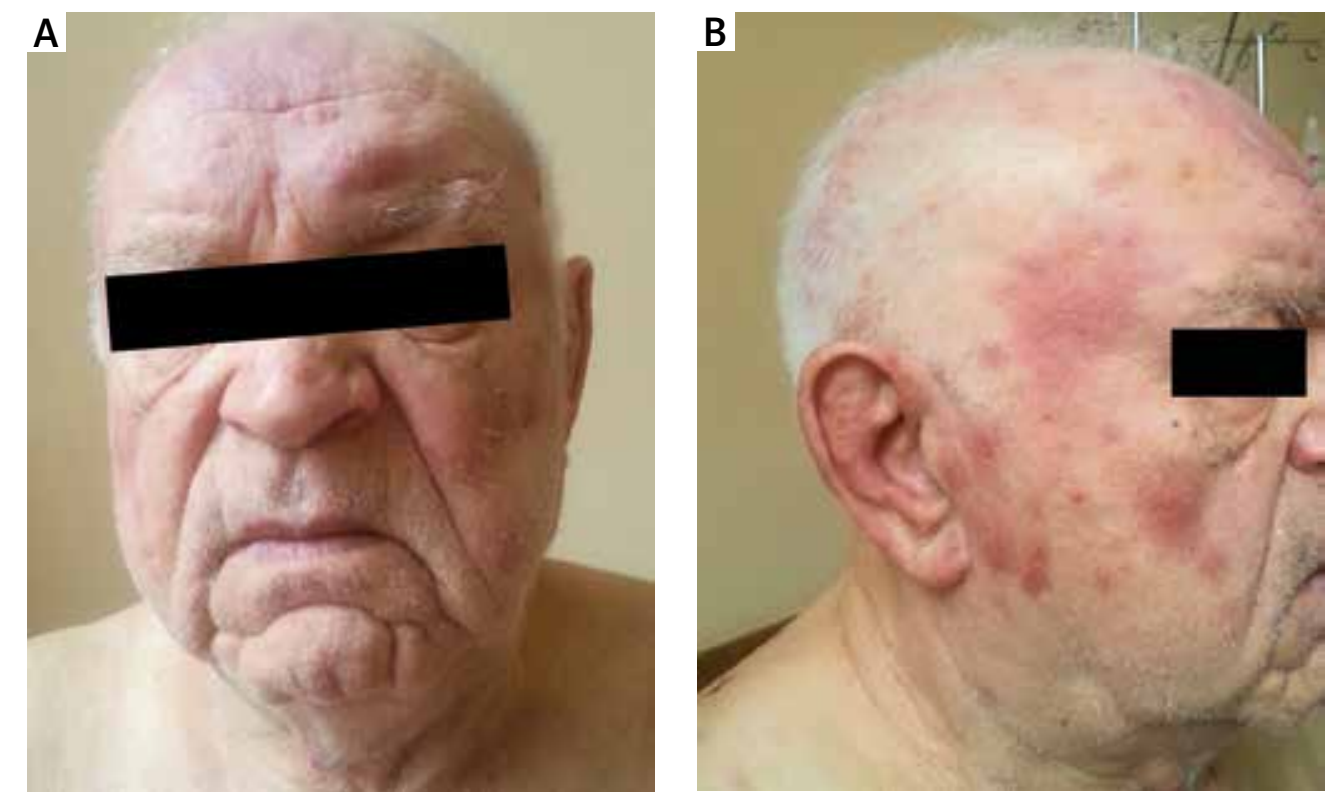

Figure 11. The patient after therapy - remission of skin lesions (A, B)
The average age of presented cases was similar to previous reports (median: 68.5 years). Literature data indicate the male to female ratio for incidence of approximately $1: 2$ to $7.25[2,7,9,10,12]$. However, there was female predominance in our group of patients.

Blastic plasmacytoid dendritic cell neoplasm shows many diagnostic dilemmas. Diagnosis is based primarily on manifested skin lesions and characteristic immunoprofiles CD4 and CD56. Frequently encountered positive markers include CD2, CD7, CD38, CD33, CD43, CD45, CD123, RA CD117, CD68, TCL 1 Bcl 11A, CLA, TDT and MXA $[20,21]$. Blastic plasmacytoid dendritic cell neoplasm cells expression of CD123, which occurs in $100 \%$ of patients, confirms that the neoplasm is derived

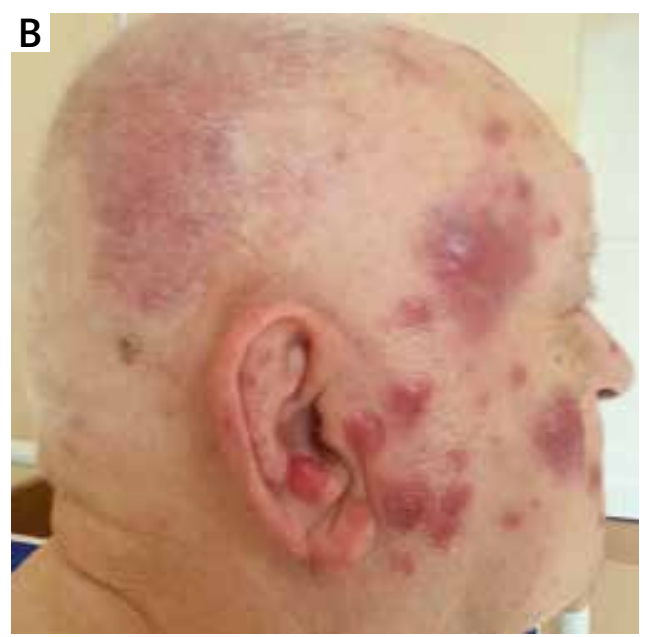

Figure 11. The patient after therapy - remission of skin lesions $(A, B)$ 
A. Owczarczyk-Saczonek, M. Sokołowska-Wojdyło, B. Olszewska, M. Malek, A. Znajewska-Pander, A. Kowalczyk, W. Biernat, G. Poniatowska-Broniek, W. Knopińska-Posłuszny, Z. Kozielec, R. Nowicki, W. Placek

Table 1. The clinical pictures and immunophenotypes of skin biopsies

\begin{tabular}{|c|c|c|c|c|c|c|c|c|c|c|c|c|c|c|c|}
\hline \multirow[t]{2}{*}{ Patient } & \multirow[t]{2}{*}{ Sex/age } & \multirow[t]{2}{*}{ Cutaneous lesion } & \multicolumn{10}{|c|}{ Immunophenotype } & \multicolumn{3}{|c|}{ Staging } \\
\hline & & & CD3 & CD4 & CD56 & TIA1 & $\begin{array}{l}\text { Granzyme } \\
\text { B1 }\end{array}$ & CD20 & CD8 & CD34 & TdT & Ki67 & LN & BM & PB \\
\hline 1 & $\mathrm{~F} / 72$ & $\begin{array}{c}\text { Face }+ \text { trunk } \\
\text { Nodular, bruise-like patches }\end{array}$ & - & + & + & - & - & - & - & - & $\begin{array}{l}-/+ \\
15 \%\end{array}$ & $70 \%$ & + & - & - \\
\hline 2 & $F / 60$ & $\begin{array}{c}\text { Trunk + extremites } \\
\text { Nodular, bruise-like patches, } \\
\text { lichenoid eruption }\end{array}$ & - & + & + & - & - & - & - & - & - & $18 \%$ & ND & + & + \\
\hline 3 & $F / 63$ & Right leg, nodules & - & + & + & ND & ND & - & ND & - & - & $85 \%$ & + & - & - \\
\hline 4 & M/79 & $\begin{array}{c}\text { Head }+ \text { face }+ \text { trunk } \\
\text { Nodular, bruise-like patches }\end{array}$ & - & + & + & ND & ND & ND & - & - & ND & ND & - & ND & + \\
\hline
\end{tabular}

F-female, $M$ - male.

from plasmacytoid dendritic cells type II, while the expression of terminal deoxynucleotidyl transferase (TdT) indicates a precursor lymphoid cell origin $[10,16]$. In our cases, immunohistochemical stains were positive for CD4 and CD56 (Table 1). Unfortunately, staining for CD123 has not been done, although it is believed to be the most characteristic marker for BPDCN, even if expression of CD4 and CD56 is weak $[2,10]$.

The tumor cells are characterized by monomorphic population with a dispersed or spheroidal pattern. On the other hand BPDCN infiltrates the dermis but spares the epidermis (no epidermotropism), Grenz zone and the adnexal structures [2, 5]. In our cases, all of the biopsies revealed typical features of BPDCN; however, patient 4 also showed fibrotic changes in collagen.

Garnache-Ottou et al. proposed the diagnostic algorithm for BPDCN. The co-expression of CD4+, CD56+, CD123+/-, BDCA2+ and/or BDCA4+ and absence of CD3, CD11C-, MPO- and CD79a- are diagnostic for BPDCN. If CD123 expression is negative or dim, or when CD123 is positive but cells do not express BDCA2 or BDCA4, then a diagnosis of BPDCN should not be considered $[5,17]$. Julia et al. analyzed 91 patients and identified 5 most characteristic immunophenotypic markers: CD4, CD56, CD123, CD303 and TCL1. Simultaneous expression of all markers was observed in $46 \%$ of patients, but the expression of 4 markers was sufficient for a diagnosis $[5,7]$.

Blastic plasmacytoid dendritic cell neoplasm must be differentiated from acute myeloid leukemia, extra nodal NK/T cell lymphoma, nasal type and certain $T$ cell lymphoma, particularly the $\gamma / \delta$ type. Certain myelomonocytic neoplasms may involve proliferation of plasmacytoid dendritic cells, but these are mature and CD56 negative [20]. Extramedullary myeloid sarcoma (EMS) may be difficult to differentiate because T-cells and NK-cells present CD4+ or CD56+ immunophenotype and both diseases frequently manifest with skin infiltration [5].

Researchers debate whether tumor cells are primarily positioned in the bone marrow or skin. The question is whether skin involvement is a consequence of cutaneous tropism of blast cells or whether it is coexistence of two different CD4+, CD56+ malignancies [22]. Currently, as mentioned above, BPDCN is qualified to acute myeloid leukemia notable for highly aggressive behavior with cutaneous, lymph node and bone marrow involvement [18].

Blastic plasmacytoid dendritic cell neoplasm has a predilection to the skin and is related to $70-85 \%$ of cases [2, 10, 23]. Cutaneous lesions seldom appear during the follow-up period [2]. In all our cases, skin lesions were the initial cause of visiting a medical doctor. The most common skin localization is the head, face and upper trunk. Patient 3 showed rare localization of skin lesions on the lower extremity, though this has been already reported in the literature [24].

Initially, the disease can be confused with drug-induced skin lesions, lupus erythematosus and eczema. Skin lesions present as nodules, tumors, erythematous plaques and bruise-like patches. The plaque diameter ranges from a few millimeters to a few centimeters, the colour ranges from dark red to characteristic purple, and ulcers occur occasionally [6, 9, 24]. Skin lesions are either isolated or may disseminate all over the body [2, $5,7,23,24]$. In the largest study, Julia et al. analyzed 91 patients who presented nodular lesions (73\%), "bruiselike' patches (12\%) and disseminated lesions (patches and nodules) (14\%) [7]. They identified three major clinical presentations: nodular lesions, bruise-like patches and mixed lesions [7, 9]. In contrast, Cota et al. analyzed 33 patients, paying attention to the distribution of lesions: patients presented with generalized $(n=18)$; localized $(n=6)$ or solitary $(n=9)$ macules, plaques, tumors [24]. Kim et al. analyzed seven patients ( 6 males and 1 female) with skin manifestations: bruise-like tumefaction $(n=6)$, erythematous nodules $(n=4)$, or multiple erythematous papules $(n=1)$, only 1 patient did not present skin involvement [2]. However, there is no correlation between the type of cutaneous clinical presentation and prognosis [9]. 
Our patients developed a range of various skin lesions that required differentiation with dermatological diseases. In those cases, histopathological and immunohistochemical examinations were needed to determine diagnosis of BPDCN. An interesting phenomenon of spontaneous partial remission of tumors has been observed in the last case. However, observation of the patient was short and his further fate is unknown.

Involvement of the bone marrow and regional lymphadenopathy is seen in $40-60 \%$ of cases. Infiltration of bone marrow leads to peripheral cytopenia that result in myelodysplastic clinical picture [7, 9]. The liver, spleen, lungs and central nervous system are rarely involved [5, 16]. Interestingly, about $10-20 \%$ of BPDCN are associated with coexistent myelomonocytic leukemia [9], as well as acute myeloid leukemia and acute lymphocytic leukemia [16]. In our patients lymph node involvement was observed most frequently, but also rapid peripheral blood involvement in one case.

The clinical course of BPDCN is aggressive with rapid bone marrow and lymph node involvement, and patients exhibit an average survival time of 12-14 months [2, 5, 12, 22, 25]. Therefore, it requires the use of aggressive treatment with CHOP chemotherapy (cyclophosphamide, doxorubicin, vincristine and prednisone) with DeVIC (carboplatin, etoposide, ifosfamide, and dexamethasone) $[23,26]$. Typical initial response to treatment is effective: $70 \%$ complete response and $10 \%$ partial response, but later tumor cells become resistant [27]. Bone marrow transplantation is considered to be the most effective method of treatment. However, patients with BPDCN are often at an advanced age and are not suitable candidates for bone marrow transplantation. Better outcomes have been reported with high-dose acute leukemia-like induction chemotherapy followed by consolidation with allogeneic hematopoietic stem cell transplantation [28]. Garcia-Recio et al. described the efficacy of the treatment with one cycle of lenalidomide and celecoxib with a good response, but this regimen was discontinued due to toxicity. Although they achieved a final complete response with a much higher than expected progression-free and overall survival in an elderly patient with comorbidities [28]. In elderly patients, radiation therapy is often used for local containment. Nevertheless, the therapeutic efficacy of radiation therapy and its optimal dose remain unclear because no standard therapy has been established for this disease [23, 25, 26, 28].

Our study had several limitations. CD123 staining was not performed, also in one case TdT staining was not done. High TdT expression (> 50\%) is associated with a favorable prognosis [2]. In addition, due to a limited number of patients it is not possible to carry out statistical analysis and comparative study, but maybe it will become a part of meta-analysis.
Blastic plasmacytoid dendritic cell neoplasm (BPDCN) is a rare aggressive hematologic malignancy, primarily found in adults. Skin lesions such as erythematous infiltrates and nodules are usually the first manifestation of the disease. Therefore, in doubtful diagnostic cases dermatologists should perform histopathological and immunohistochemistry examinations along with hematological and oncological cooperation, as early diagnosis and appropriate treatment is essential for improvement of the disease course. This analysis, despite the small number of patients may provide useful information on the clinical and histopathological features of this rare malignancy.

\section{Acknowledgments}

Agnieszka Owczarczyk-Saczonek and Małgorzata Sokołowska-Wojdyło equal input into publication.

\section{Conflict of interest}

The authors declare no conflict of interest.

\section{References}

1. Bueno C, Almeida J, Lucio P, et al. Incidence and characteristics of CD4(+)/HLA DRhi dendritic cell malignancies. Haematologica 2004; 89: 58-69.

2. Kim JH, Park HY, Lee JH, et al. Blastic plasmacytoid dendritic cell neoplasm: analysis of clinicopathological feature and treatment outcome of seven cases. Ann Dermatol 2015; 27: 727-37.

3. Ng AP, Lade S, Rutherford T, et al. Primary cutaneous CD4+/ CD56+ hematodermic neoplasm (blastic NK-cell lymphoma): a report of five cases. Haematologica 2006; 91: 143-4.

4. Pagano L, Valentini CG, Pulsoni A, et al. GIMEMA-ALWP (Gruppo Italiano Malattie EMatologiche dell'Adulto, Acute Leukemia Working Party) Blastic plasmacytoid dendritic cell neoplasm with leukemic presentation: an Italian multicenter study. Haematologica 2013; 98: 239-46.

5. Riaz W, Zhang L, Horna P, Sokol L. Blastic plasmacytoid dendritic cell neoplasm: update on molecular biology, diagnosis, and therapy. Cancer Control 2014; 21: 279-89.

6. Wang W, Li W, Jia JJ, et al. Blastic plasmacytoid dendritic cell neoplasm: a case report. Oncol Lett 2015; 9: 1388-92.

7. Julia F, Petrella T, Beylot-Barry M, et al. Blastic plasmacytoid dendritic cell neoplasm: clinical features in 90 patients. Br J Dermatol 2013; 169: 579-86.

8. Maryniak RK, Jankowska-Konsur A. Zasady diagnostyki histoklinicznej i immunohistochemicznej chłoniaków pierwotnie skórnych. Pol J Pathol 2011; 1 (supl. 1): s1-22.

9. Shapiro R, Sangle N, Keeney M, et al. Blastic plasmacytoid dendritic cell neoplasm: a review of diagnosis, pathology, and therapy. J Cell Sci Ther 2015; S8: 008.

10. Petrella T, Bagot M, Willemze R, et al. Blastic NK-cell lymphomas (agranular CD4+CD56+ hematodermic neoplasms): a review. Am J Clin Pathol 2005; 123: 662-75.

11. Zhang YW, Zhong JH, Chen XL, et al. Blastic plasmacytoid dendritic cell neoplasm: a case report and literature review. Exp Ther Med 2016; 12: 319-22. 
12. Shi Y, Wang E. Blastic plasmacytoid dendritic cell neoplasm: a clinicopathologic review. Arch Pathol Lab Med 2014; 138: 564-9.

13. Willemze R, Jaffe ES, Burg G, et al. WHO-EORTC classification for cutaneous lymphomas. Blood 2005; 105: 3768-85.

14. Facchetti F, Jones DM, Petrella T. Blastic plasmacytoid dendritic cell neoplasm. In: WHO Classification of Tumors of Haematopoietic and Lymphoid Tissues, Swerdlow SH, Campo E, Harris NL, et al. (eds.), IARC, Lyon 2008; 145.

15. Fenillard J, Jacob MC, Valensi F, et al. Clinical and biologic features of CD4 CD56 malignancies. Blood 2002; 99: 1556-63.

16. Pemmaraju N. Blastic plasmacytoid dendritic cell neoplasm. Clin Adv Hematol Oncol 2016; 14: 220-2.

17. Garnache-Ottou F, Feuillard J, Ferrand C, et al. Extended diagnostic criteria for plasmacytoid dendritic cell leukaemia. Br J Haematol 2009; 145: 624-36.

18. Andrese E, Solovăstru LG, Dimofte G, et al. Blastic plasmacytoid dendritic cell neoplasm - a rapidly evolving entity. Case report. Rev Med Chir Soc Med Nat lasi 2015; 119: 379-83.

19. Mizia-Malarz A, Sobol-Milejska G. Blastic plasmocytoid dendritic cell neoplasm with skin and myeloid location. J Pediatr Hematol Oncol 2018; 40: e29-e31.

20. Arora R, Sachdev R, Bind T, Khan MA. Blastic plasmacytoid dendritic cell neoplasm: a report of 2 cases. Indian J Hematol Blood Transfus 2013; 29: 171-2.

21. Facchetti F, Ungari M, Marocolo D, et al. Blastic plasmacytoid dendritic cell neoplasm. Hematol Meet Rep 2009; 3: 1-3.

22. Lencastre A, Cabete J, João A, et al. Blastic plasmacytoid dendritic cell neoplasm. An Bras Dermatol 2013; 88 (6 Suppl. 1): 158-61.

23. Ishibashi N, Maebayashi T, Aizawa T, et al. Radiation therapy for cutaneous blastic plasmacytoid dendritic cellneoplasm: a case report and review of the literature. Int I Clin Exp Med 2015; 8: 8204-9.

24. Cota C, Vale E, Viana I, et al. Cutaneous manifestations of blastic plasmacytoid dendritic cell neoplasm-morphologic and phenotypic variability in a series of $33 \mathrm{pa}-$ tients. Am J Surg Pathol 2010; 34: 75-87.

25. Xin X, Tian M, Huang L, et al. Case report cutaneous lesions from a blastic plasmacytoid dendritic cell neoplasm: a case report and literature review. Int J Clin Exp Med 2016; 9: 5017-22.

26. Dalle S, Beylot-Barry M, Bagot M, et al. Blastic plasmacytoid dendritic cell neoplasm: is transplantation the treatment of choice? Br J Dermatol 2010; 162: 74-9.

27. Saeed H, Awasthi M, Al-Qaisi A, Massarweh S. Blastic plasmacytoid dendritic cell neoplasm with extensive cutaneous and central nervous system involvement. Rare Tumors 2014; 6: 5474.

28. Garcia-Recio M, Martinez-Serra J, Bento L, et al. Lenalidomide, celecoxib, and azacitidine therapy for blastic plasmocytoid dendritic cell neoplasm: a case report. Onco Targets Ther 2016; 9: 5507-11. 